\title{
Efficacy of limited hippocampal radiofrequency thermocoagulation for mesial temporal lobe epilepsy
}

\author{
Ching-Yi Lee, MD, ${ }^{1}$ Han-Tao Li, MD, ${ }^{2}$ Tony Wu, MD, PhD, ${ }^{2}$ Mei-Yun Cheng, MD, PhD, ${ }^{2}$ \\ Siew-Na Lim, MD, PhD, ${ }^{2}$ and Shih-Tseng Lee, MD' \\ 'Department of Neurosurgery; and 'Section of Epilepsy, Department of Neurology, Chang Gung Memorial Hospital Linkou \\ Medical Center and Chang Gung University College of Medicine, Taoyuan, Taiwan
}

\begin{abstract}
OBJECTIVE Radiofrequency thermocoagulation (RFTC), which has been developed for drug-resistant epilepsy patients, involves less brain tissue loss due to surgery, fewer surgical adverse effects, and generally good seizure control. This study demonstrates the effectiveness of RFTC performed at limited hippocampal locations.

METHODS Daily seizure diaries were prospectively maintained for at least 6 months by 9 patients (ages 30-59 years) with drug-resistant mesial temporal lobe epilepsy (MTLE) before treatment with RFTC. The limited target for stereotactic RFTC was chosen based on intraoperative electroencephalography (EEG) recording and was initially tested with a Radionics electrode at a low temperature, $45^{\circ} \mathrm{C}$, for 60 seconds. The therapeutic RFTC heating parameters were $78^{\circ} \mathrm{C}-80^{\circ} \mathrm{C}$ for 90 seconds. All patients who received the RFTC treatment underwent both MRI and EEG recording immediately postoperatively and at the 3-month follow-up. Monthly outpatient clinic visits were arranged over 6 months to document seizure frequency and severity to clarify the changes noted in imaging studies and EEG patterns.
\end{abstract}

RESULTS Two patients were excluded from our analysis because one had undergone multiple seizure surgeries and the other had a poor recording of seizure frequency, before the RFTC surgery. Five and two patients underwent left-sided and right-sided RFTC, respectively. None of the patients had generalized tonic-clonic attacks postoperatively, and no adverse effects or complications occurred. According to MRI data, the effect of coagulation was limited to less than 1.0 $\mathrm{cm}$ in diameter and perifocal edema was also in limited range. The seizure frequency within 6 months decreased postoperatively with a mean reduction in seizures of $78 \%$ (range $36 \%-100 \%$ ). Only two patients had a temporary increase in seizure frequency within 2 weeks of the surgery, and over $50 \%$ of all patients showed a decrease in average seizure frequency.

CONCLUSIONS The study results confirm that limited RFTC provides a more effective surgery with similar seizure control but fewer complications than resective surgery for drug-resistant MTLE patients.

https://thejns.org/doi/abs/10.3171/2018.4.JNS184

KEYWORDS radiofrequency thermocoagulation; drug-resistant mesial temporal lobe epilepsy; stereoelectroencephalography; minimally invasive epilepsy surgery

$\mathrm{E}$ PILEPSY has an annual prevalence of $1 \%$ of the population, and approximately $30 \%$ of patients continue to experience seizures despite medication. Although decades of targeted drug discovery have yielded improved side-effect profiles, there has been little progress in decreasing the number of patients with medication-resistant epilepsy. ${ }^{12}$ Mesial temporal lobe epilepsy (MTLE), the most common focal epilepsy in adults secondary to hippo- campal sclerosis or gliosis, is often resistant to pharmacotherapy and requires surgical intervention, which typically results in favorable outcomes. ${ }^{5,7,11,22}$ Although Wiebe et al. ${ }^{22}$ reported that patients with MTLE who had undergone resective surgery had good seizure control, these authors documented significant postoperative memory impairment as well. ${ }^{18}$ Consequently, minimally invasive epilepsy surgery such as stereotactic radiosurgery, radiofrequency

ABBREVIATIONS AED = antiepileptic drug; CPS = complex partial seizure; $D G$ = dentate gyrus; EEG = electroencephalography; GTC = generalized tonic-clonic; $M$ TLE = mesial temporal lobe epilepsy; RFTC = radiofrequency thermocoagulation; SEEG = stereo-EEG.

SUBMITTED January 2, 2018. ACCEPTED April 26, 2018.

INCLUDE WHEN CITING Published online October 26, 2018; DOI: 10.3171/2018.4.JNS184. 
thermocoagulation (RFTC), laser-induced thermal therapy, and MRI-guided focused ultrasound ablation was developed, resulting in less brain tissue loss due to surgery, fewer surgery-related adverse effects, and generally good seizure control. ${ }^{16}$

The effectiveness of RFTC performed at limited locations has not been previously reported. In the current study, we present details on a minimum 6-month followup of drug-resistant MTLE patients following RFTC and identify the seizure control rate and the postoperative complications for this limited procedure.

\section{Methods \\ Subjects and Study Design}

The study protocol was approved by the Institutional Ethics Board of Chang Gung Memorial Hospital, Linkou, Taiwan. Nine patients with drug-resistant unilateral MTLE were enrolled in the study, and informed consent from all patients was obtained. These patients were selected based on the following criteria: 1) suspicion of unilateral MTLE on the basis of video-electroencephalography (EEG) monitoring, 2) ability to keep a reliable seizure diary and a prospective seizure frequency of at least two complex partial seizures (CPSs) per month or secondary generalized tonic-clonic (GTC) seizures during a baseline of 3 months, and 3) failed response to three or more antiepileptic drugs (AEDs) and currently receiving one to three AEDs. Resective surgery has been one of the treatment options for such patients in the past; however, memory outcome after temporal lobe surgery varies with the extent of the removal of nonlesional functional mesial tissues. ${ }^{17}$ Stereotactic RFTC with a limited target size was chosen for these nine patients given concerns about reducing the hospital length of stay, immediate effects on postoperative EEG, ${ }^{13}$ possible significant worsening of memory postoperatively, the presence of unilateral hippocampal sclerosis, or suspected unilateral epileptogenic zones.

Preoperative workup consisted of careful description of the seizures, neurological examinations, AED blood levels, and serial EEG including video-EEG, brain MRI, brain FDG-PET, and/or SPECT. Before RFTC surgery, daily seizure diaries were prospectively maintained for at least 6 months, and the average seizure frequency served as baseline seizure data, which were compared with seizure data after RFTC surgery. Potential adverse events were closely monitored. All AEDs remained unchanged within the first 6 months after operation; however, they could be subsequently adjusted to minimize side effects or to achieve seizure control.

\section{Surgical Procedures}

For better intraoperative recording of epileptic discharges, local anesthesia was chosen and all AEDs were withheld 12 hours before operation to minimize the possibility of a poor recording of epileptic origins during the procedure. With the patient under local anesthesia, a Brown-Roberts-Wells stereotactic frame was applied and thin-slice brain CT was performed for preoperative localization of the target side of the anterior portion of the hippocampus. After localization, an electrode (deep brain stimulation lead model 3389s, Medtronic) was inserted via a parasagittal occipital approach with the patient in a sitting position for intraoperative EEG recording from the target side of the hippocampus. The EEG recordings obtained from four contact points revealed spike-and-wave complexes over different lead locations; this indicated that the presumed epileptogenic focus of the hippocampus was accurately targeted. If the spike-and-wave complexes continued until the fourth lead, the leads were withdrawn until no further detection of spike-and-wave complexes. The electrode was then removed and changed to a Radionics (Cosman Medical Inc.) radiofrequency lead inserted via the same trajectory. The target and duration of the RFTC were chosen according to the results of the intraoperative recording. The safety of thermocoagulation was tested at a low temperature, $45^{\circ} \mathrm{C}$, for 60 seconds for any sign of adverse effects. We then changed to the therapeutic RFTC heating parameters of $78^{\circ} \mathrm{C}-80^{\circ} \mathrm{C}$ for 90 seconds. The lead was then removed, and the wound was sutured. The location of thermocoagulation was further confirmed by postoperative brain MRI. Immediate postoperative EEG was also performed to see the changes in the seizure pattern or epileptic discharge waveform after the RFTC intervention.

\section{Follow-Up Design}

All patients treated with RFTC surgery underwent both MRI and EEG recording immediately after the operation. Monthly outpatient clinic visits were arranged to occur over at least 6 months postoperatively to record seizure frequency and severity as well as seizure outcome as assessed using the Engel classification. ${ }^{6}$ Preoperative and postoperative neuropsychological examinations were performed using the Wechsler Adult Intelligence Scale ${ }^{21}$ to evaluate memory function and IQ. Follow-up brain MRI and video-EEG were performed at 3 months after surgery to clarify changes in the imaging studies and EEG patterns.

\section{Results}

Nine patients underwent RFTC surgery at Chang Gung Memorial Hospital Linkou; however, one of them was excluded because multiple seizure surgeries including deep brain stimulation had been performed before the RFTC treatment, and another one was excluded because of poor recording of seizure frequency before the RFTC surgery. In total, three male and four female patients were included in our analysis (Table 1). The mean age of these patients was 42.7 years. Two patients had right-sided MTLE and the other five had left-sided MTLE. The average age at disease onset was 21.3 years, and the patients had experienced seizure attacks for an average of 21.4 years. The average follow-up period after surgery was 12.6 months, and no adverse effects of surgery were noted. Five patients had left-sided RFTC and two had right-sided RFTC. On preoperative MRI examination, only one patient had hippocampal sclerosis. Five of the seven patients had GTC attacks before surgery, and none had GTC attacks after surgery. The two patients without GTC attacks before RFTC did experience consciousness disturbance or 
TABLE 1. General characteristics of 7 patients who underwent RFTC

\begin{tabular}{|c|c|c|c|c|c|c|c|c|c|c|c|c|}
\hline $\begin{array}{l}\text { Case } \\
\text { No. }\end{array}$ & Sex & $\begin{array}{c}\text { Age at } \\
\text { RFTC } \\
\text { (yrs) }\end{array}$ & $\begin{array}{c}\text { Age at } \\
\text { Disease } \\
\text { Onset (yrs) }\end{array}$ & $\begin{array}{c}\text { Duration } \\
\text { of Szs } \\
\text { (yrs) }\end{array}$ & $\begin{array}{l}\text { Lesion } \\
\text { Side }\end{array}$ & $\begin{array}{c}\text { FU } \\
(\mathrm{mos})\end{array}$ & $\begin{array}{c}\text { Postop } \\
\text { Adverse } \\
\text { Effect }\end{array}$ & $\begin{array}{c}\text { Intraop } \\
\text { Sz } \\
\text { Attack }\end{array}$ & $\begin{array}{c}\text { GTC/CPS } \\
\text { Before } \\
\text { RFTC }\end{array}$ & $\begin{array}{c}\text { GTC/CPS } \\
\text { After } \\
\text { RFTC }\end{array}$ & $\begin{array}{c}\text { Hippocampal } \\
\text { Sclerosis }\end{array}$ & $\begin{array}{c}\text { Surgical } \\
\text { Adverse } \\
\text { Event }\end{array}$ \\
\hline 1 & $F$ & 37 & 23 & 14 & $\mathrm{Lt}$ & 24 & $\mathrm{~N}$ & $Y$ & $Y / Y$ & $\mathrm{~N} / \mathrm{N}$ & $\mathrm{N}$ & $\mathrm{N}$ \\
\hline 2 & $F$ & 37 & 3 & 34 & $\mathrm{Rt}$ & 21 & $\mathrm{~N}$ & $\mathrm{~N}$ & $Y / Y$ & $N / Y^{*}$ & $\mathrm{~N}$ & $\mathrm{~N}$ \\
\hline 3 & $M$ & 59 & 18 & 41 & $\mathrm{Lt}$ & 10 & $\mathrm{~N}$ & $\mathrm{~N}$ & $Y / Y$ & $\mathrm{~N} / \mathrm{N}$ & $\mathrm{N}$ & $\mathrm{N}$ \\
\hline 4 & $M$ & 30 & 24 & 6 & $\mathrm{Rt}$ & 10 & $\mathrm{~N}$ & $\mathrm{~N}$ & $Y / Y$ & $N / Y$ & $\mathrm{~N}$ & $\mathrm{~N}$ \\
\hline 5 & $F$ & 33 & 10 & 23 & $\mathrm{Lt}$ & 9 & $\mathrm{~N}$ & $\mathrm{~N}$ & $N / Y$ & $\mathrm{~N} / \mathrm{N}$ & $\mathrm{N}$ & $\mathrm{N}$ \\
\hline 6 & $F$ & 47 & 39 & 8 & $\mathrm{Lt}$ & 7 & $\mathrm{~N}$ & $Y$ & $N / Y$ & $\mathrm{~N} / \mathrm{N}$ & $Y$ & $\mathrm{~N}$ \\
\hline 7 & $M$ & 56 & 32 & 24 & $\mathrm{Lt}$ & 7 & $\mathrm{~N}$ & $Y$ & $Y / Y$ & $\mathrm{~N} / \mathrm{N}$ & $\mathrm{N}$ & $\mathrm{N}$ \\
\hline Mean & & 42.7 & 21.3 & 21.4 & & 12.6 & & & & & & \\
\hline
\end{tabular}

$\mathrm{FU}=$ follow-up; $\mathrm{N}=$ no; $\mathrm{Sz}=$ seizure; $\mathrm{Y}=$ yes.

Each patient had a hospital stay of 4 days.

${ }^{*}$ Decreased frequency.

memory impairment related to CPS attacks before surgery, but the consciousness disturbance did not occur after the operation. Three patients had minor seizure attacks or aura sensation during the operation mostly at the time of high-temperature coagulation, but no adverse effect or complication occurred during or after the operation, such as postoperative bleeding or infection. Postoperative MRI was performed immediately after the procedure to identify the lesion site of the RFTC target and confirm location accuracy (Fig. 1). According to the MRI data, the effect of coagulation was limited to less than $1.0 \mathrm{~cm}$ in diameter, and perifocal edema also appeared within a limited range. The sizes of the RFTC targets according to MRI are listed in Table 2. According to the results, the effect of thermocoagulation was very much limited to a certain area of the hippocampus, and no additional trauma was caused by the procedure. The seizure frequency change over the 6 months after the operation is shown in Fig. 2, and the Engle classification ${ }^{6}$ of seizure control outcome is listed in Table 3. The results of preoperative and postoperative neuropsychological examination are shown in Table 4 and reveal no significant change in memory function and IQ after the procedure. As shown in Fig. 2, the average seizure attacks decreased in frequency during the 6-month follow-up with a mean reduction in seizures of 78\% (range $36 \%-100 \%$ ). Only two patients had a temporary increase in seizure frequency by 2 weeks after RFTC, and over $50 \%$ of all the patients showed a reduction in seizure frequency.

\section{Illustrative Case 1}

The following illustrative case has been reprinted in part from World Neurosurgery, 96, Li et al., Seizure freedom after limited hippocampal radiofrequency thermocoagulation, 612.e21-612.e25, Copyright 2016, with permission from Elsevier. Text that does not appear in the original work has been placed in brackets.

\section{History and Examination}

A 37-year-old, right-handed woman had febrile convulsions since the age of 3 . She first presented to our neurologic clinic for [GTC] seizure at age $23 .{ }^{13}$ She received AED treatment, consisting of valproate $400 \mathrm{mg} /$ day and topiramate $200 \mathrm{mg} /$ day. Following AED treatment, the [GTC] seizure was under control. However, she noted another seizure pattern at age 23 , which manifested with an aura of an epigastric rising sensation, immediately followed by nonresponsive consciousness, sustained gaze deviation to the right, speechlessness, right arm automatism, and postictal confusion. Seizure durations were typically 1 minute. Noted trigger factors were menstrual cycle, anxiety, sleep deprivation, and the common cold. AED treatment was gradually changed to oxcarbazepine $450 \mathrm{mg}$ /day. . . .

Reviewing her medical history, she had normal developmental milestones and reported no history of central nervous system infection, head injury, status epilepticus, autoimmune disease, or family history of epilepsy. Her neurologic and general physical examinations revealed normal results. Routine EEG showed interictal epileptiform discharges over the left frontotemporal area. MRI of the brain showed left hippocampal atrophy. Neuropsychological tests revealed normal mental function, . . . and full IQ of 96.

The patient's seizure frequency increased to 5-18 times per 12-week period in the 4 years before surgery, despite an increase in oxcarbazepine dosage to $1200 \mathrm{mg}$ /day 3 years before surgery. Long-term video-EEG monitoring revealed frequent solitary spikes in the left temporal region with F7-T1 phase reversal. One habitual event initially manifested with a lapse of consciousness, head turning to the right side, and tonic twitching of the limbs for a duration of 1 minute. The concurrent EEG findings demonstrated rhythmic theta from F7-T1 that propagated to the left frontotemporal area [Fig. 3]. The patient was diagnosed with drug-resistant left MTLE, which was compatible with preoperative MRI findings. Finding clear epileptogenic foci, we planned to perform [limited] RFTC over the left hippocampus. [Because of poor medical control of her seizures, RFTC was arranged, and intraoperative EEG recording assistance was used to identify the location of possible seizure foci (Fig. 4).] . . .

\section{Postoperative Course}

The AED treatment of oxcarbazepine $1200 \mathrm{mg}$ mono- 

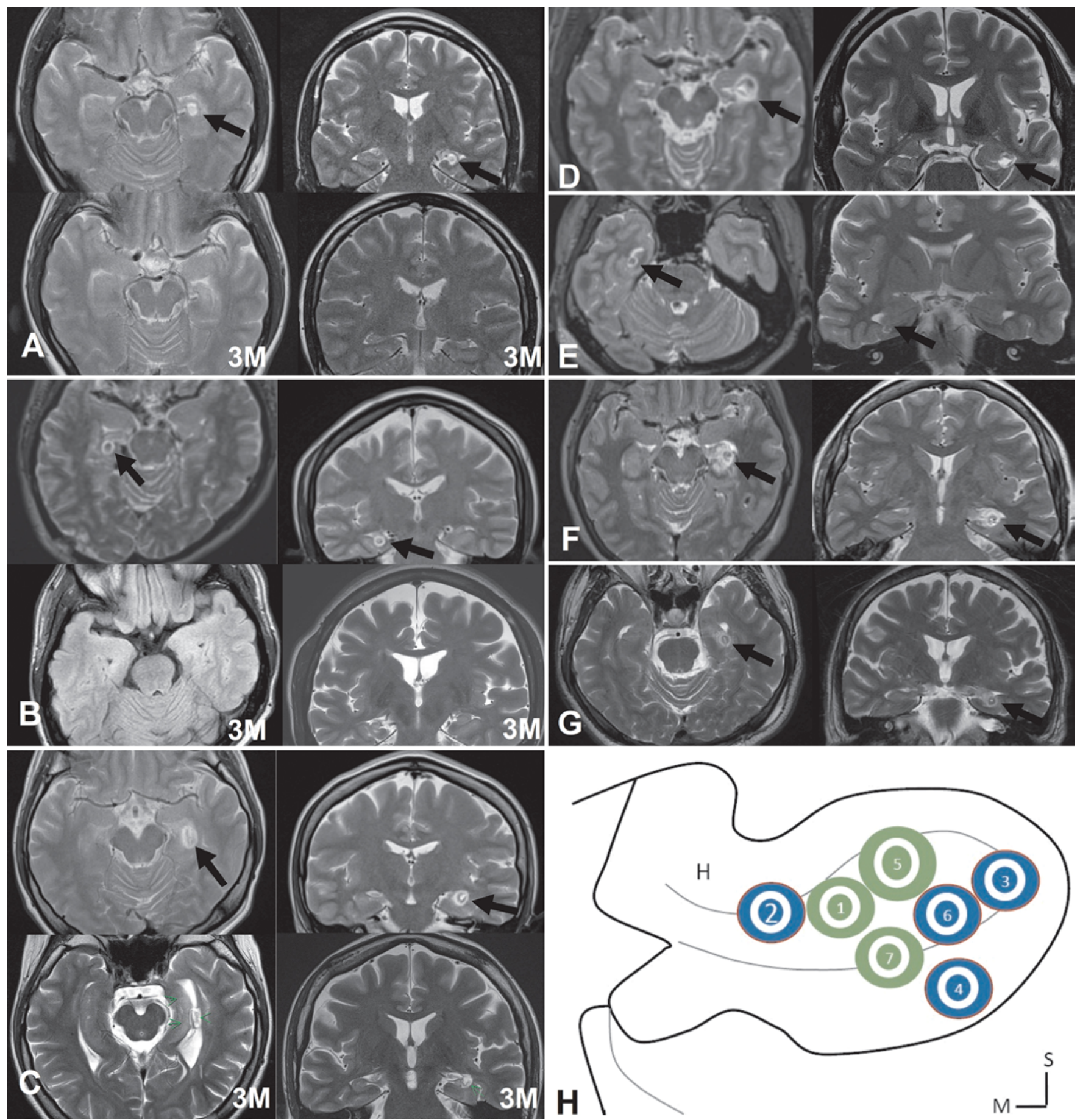

FIG. 1. Immediately postoperative (upper) axial (left) and coronal (right) T2-weighted MR images indicate limited sites for the RFTC lesions (arrows) in cases 1 (A), 2 (B), and 5 (C). Three-month postoperative (lower) axial (left) and coronal (right) MR images reveal resolution of the thermocoagulation lesions with further reduction of perilesional edema in cases 1,2 , and 5 , respectively. Immediately postoperative axial (left) and coronal (right) T2-weighted MR images show limited sites for the RFTC lesions (arrows) in cases $3(\mathbf{D}), 4(\mathbf{E}), 6(\mathbf{F})$, and $7(\mathbf{G})$. Note the correlation $(\mathbf{H})$ of seizure control in each case (indicated by numbers) with the RFTC lesion size and the location of the hippocampal lesion site. Cases 1, 5, and 7 (green), whose lesion sites were closer to the dentate gyrus, had a better seizure control outcome than cases $2-4$ and 6 (blue), who had relatively worse seizure control. $\mathrm{H}=$ hippocampus; $\mathrm{M}=$ medial; $\mathrm{S}=$ superior; $3 \mathrm{M}=3$-month. Figure is available in color online only. 
TABLE 2. Sizes of the RFTC targets according to MRI in the 7 patients

\begin{tabular}{cccccc}
\hline Case No. & RFTC Side & RFTC Vol $\left(\mathrm{mm}^{3}\right)$ & Lesioned Hippocampus Vol $\left(\mathrm{mm}^{3}\right)$ & Nonlesioned Hippocampus Vol $\left(\mathrm{mm}^{3}\right)$ & Lesioned/Nonlesioned Vol Ratio \\
\hline 1 & $\mathrm{Lt}$ & 380.5 & 1213.4 & 2327.1 & 0.314 \\
\hline 2 & $\mathrm{Rt}$ & 224.4 & 1837.8 & 2132 & 0.122 \\
\hline 3 & $\mathrm{Lt}$ & 591.9 & 1794.6 & 1202 & 0.330 \\
\hline 4 & $\mathrm{Rt}$ & 168.4 & 2909.8 & 2349.5 & 0.039 \\
\hline 5 & $\mathrm{Lt}$ & 621 & 1973.5 & 2335 & 0.315 \\
\hline 6 & $\mathrm{Lt}$ & 212 & 2350.2 & 2450 & 0.09 \\
\hline 7 & $\mathrm{Lt}$ & 243 & 2349 & 2775 & 0.10 \\
\hline Mean & & 348.74 & 2061.18 & 2224.37 & 0.187 \\
\hline
\end{tabular}

therapy was unchanged after surgery, and the patient remained seizure-free for [2 years] after treatment except for 1 minor simple partial seizure at week 18. Postoperative MRI performed 2 months later revealed resolved perilesional edema of the previous coagulated lesion; only a 3-mm T2 hyperintensity area was noted, which corresponded to the center of the coagulated area [Fig. 1A]. Follow-up video-EEG monitoring for 3 hours showed a normal background with infrequent F7-T1 reversal. There were no side effects related to the surgical procedure or symptoms of memory impairments throughout the followup period.

\section{Discussion}

The results of this study suggest that limited RFTC at the hippocampus can be safe and effective for patients with drug-resistant MTLE. Comparisons of seizure frequency at baseline and during the postoperative period revealed a mean reduction in seizures of $78 \%$ (range 36\%-100\%) in 7 patients, including 1 patient with MRI evidence of hippocampal sclerosis. Although Cossu et al. ${ }^{3}$ reported the results of RFTC performed in 89 patients, the target of RFTC was multifocal, namely the amygdala, temporal pole, and basal temporal cortex, and the diagnosis in their patients was different from MTLE. To the best of our knowledge, our study is the first to show that patients with drug-resistant MTLE who undergo limited hippocampal RFTC experience a decrease in seizure frequency by the long-term follow-up. Our results suggest that hippocampal RFTC should be considered for patients with drug-resistant MTLE given its effectiveness in controlling seizure frequency and severity.

Radiofrequency thermocoagulation for epileptic foci was introduced in 2004, ${ }^{10}$ when investigators used stereoEEG (SEEG)-guided RFTC to treat 20 patients with different locations of seizure foci and revealed that the procedure may be safe and lead to a significant reduction in seizure frequency in well-selected patients. Also, Dimova et al. ${ }^{4}$ reported that $\mathrm{RFTC}$ can be a reasonable therapeutic option in complex cases in which resective surgery may be unsuitable, in an attempt to preserve the neurological functional outcome, especially when surgery is associated with an anatomical location such as the hippocampus. Thus, patients with drug-resistant MTLE can consider RFTC a treatment option that causes less destruction of the hippocampus and less damage to memory and cogni- tive function as compared to those following traditional hippocampectomy. Furthermore, patients treated with RFTC have a shorter hospital stay than those treated with traditional hippocampectomy and show quicker recovery with smaller wounds because of the less destructive procedure. Consequently, RFTC has unique value for patients with drug-resistant MTLE given the comparatively low cost of the whole procedure, the short hospital stay, and the ease of incorporating the procedure by different hospitals with various resources without the need for intensive postoperative care.

The main purpose of various epilepsy surgeries is to reduce seizure frequency or the severity of each attack, and their outcomes have been researched. Regarding patients who have undergone RFTC for epilepsy control, two of the largest published series have demonstrated significant improvement in $28 \%$ of patients ${ }^{3}$ or a $\geq 50 \%$ reduction in seizure frequency in $48 \%$ of patients, ${ }^{1}$ respectively, after RFTC at various locations; seizure freedom was achieved in $18 \%$ and $7 \%$ of the patients, respectively. ${ }^{1,3}$ For patients

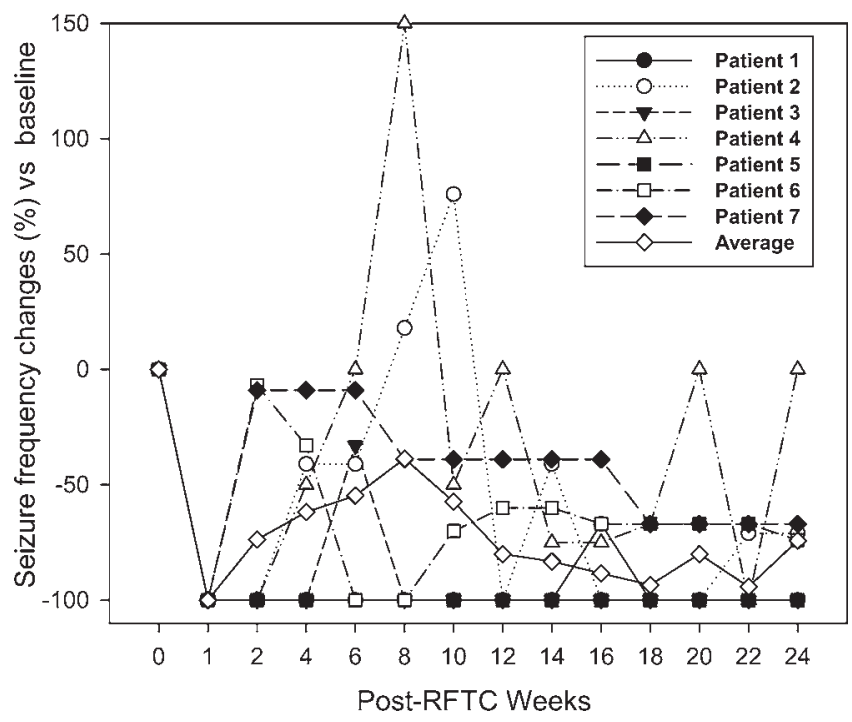

FIG. 2. Seizure frequency changes with respect to baseline over 6 months in the 7 patients after RFTC. All patients showed a decrease in seizure attacks, except for the patients in cases 2 (empty circles) and 4 (empty triangles), who had increased seizure frequency after only 2 weeks. The average reduction in seizure frequency for all patients ranged from $36 \%$ to $100 \%$ with a mean value of $78 \%$ (empty diamonds). 
TABLE 3. Seizure frequency change and results of seizure control in 7 patients over 6 months

\begin{tabular}{|c|c|c|c|c|c|c|c|c|}
\hline \multirow{2}{*}{$\begin{array}{c}\text { Case } \\
\text { No. }\end{array}$} & \multicolumn{7}{|c|}{ Sz Frequency } & \multirow{2}{*}{$\begin{array}{l}\text { Sz Control Outcome: } \\
\text { Engle Class }\end{array}$} \\
\hline & Baseline (per mo) & 1 Mo Postop & 2 Mos Postop & 3 Mos Postop & 4 Mos Postop & 5 Mos Postop & 6 Mos Postop & \\
\hline 1 & 6 & 0 & 0 & 0 & $1^{*}$ & 0 & 0 & I \\
\hline 2 & 3.4 & 1 & 3 & 3 & 1 & 2 & 2 & III \\
\hline 3 & 2 & 0 & 1 & 0 & 0 & 0 & 0 & II \\
\hline 4 & 4 & 0 & 5 & 3 & 1 & 1 & 4 & III \\
\hline 5 & 3.2 & 0 & 0 & 0 & 0 & 0 & 0 & I \\
\hline 6 & 30 & 20 & 0 & 12 & 10 & 10 & 8 & III \\
\hline 7 & 2.2 & 2 & 2 & 1 & 1 & 1 & 1 & II \\
\hline
\end{tabular}

${ }^{*}$ Aura only.

with epileptic origins in the hippocampus, the effects of different operations have been studied. Radiofrequency ablation surgery for drug-resistant MTLE has been reported on previously ${ }^{20}$ with a significant seizure freedom rate up to $70 \%$ at the 2-year follow-up. The seizure freedom rate with radiofrequency ablation and extended resective surgery at the hippocampus was compared and showed no significant difference between the two $(75.6 \%$ and $76.5 \%$, respectively). ${ }^{14}$ In our current report, $57 \%$ of the patients had seizure freedom and an average seizure frequency reduction rate of $78 \%$ during a 6-month follow-up period. Although reported seizure freedom rates vary in different studies because of the different locations targeted by RFTC and the number of thermocoagulations performed, all reported seizure reduction rates have been significant. As stated above, a seizure freedom rate of only about $57 \%$ was observed in our study, but none of our patients reported a major or GTC seizure attack after RFTC performed at the hippocampus. The ideal purpose of limited RFTC at the hippocampus is to stop the vicious cycle of seizure attacks when abnormal electrical discharges originate from the mesial temporal lobe, and the ideal target for RFTC should be the dentate gyrus (DG) to avoid further propagation of epileptic discharges. As Fig. 1H illustrates, the seizure control outcome correlated better with the location of the RFTC target rather than the size of lesioning. The better epilepsy control outcome was related to the lesion site near the DG rather than the proportion of lesioned hippocampus. For patients with drug-resistant MTLE with-

TABLE 4. Preoperative and postoperative results of neuropsychological tests in the 7 patients

\begin{tabular}{crrrrrrrrrr}
\hline \multirow{2}{*}{$\begin{array}{c}\text { Case } \\
\text { No. }\end{array}$} & \multicolumn{4}{c}{ Preop } & & \multicolumn{4}{c}{ Postop } \\
\cline { 3 - 6 } \cline { 8 - 10 } & VIQ & PIQ & FSIQ & Memory & & VIQ & PIQ & FSIQ & Memory \\
\hline 1 & 97 & 99 & 98 & $8 / 10$ & & 101 & 107 & 103 & $8 / 10$ \\
\hline 2 & 78 & 68 & 73 & $2 / 10$ & & 81 & 74 & 77 & $2 / 10$ \\
\hline 3 & 83 & 77 & 80 & $3 / 10$ & & 80 & 75 & 77 & $3 / 10$ \\
\hline 4 & 108 & 97 & 104 & $10 / 10$ & & 110 & 99 & 106 & $10 / 10$ \\
\hline 5 & 84 & 95 & 87 & $8 / 10$ & & 89 & 103 & 99 & $8 / 10$ \\
\hline 6 & 111 & 106 & 109 & $7 / 10$ & & 112 & 105 & 109 & $7 / 10$ \\
\hline 7 & 101 & 99 & 101 & $9 / 10$ & & 104 & 103 & 103 & $9 / 10$ \\
\hline
\end{tabular}

$\mathrm{FSIQ}=$ Full Scale IQ; $\mathrm{PIQ}=$ Performance $I Q ; \mathrm{VIQ}=$ Verbal IQ . out lesions such as hamartoma, a precise limited target of epileptic origin or the DG can block epileptic discharges, keeping them from spreading out and inducing a further generalized effect.

Our seizure reduction rate was similar to that following radiofrequency ablation surgery, but our results showed less damage and destruction of brain parenchyma. The outcome achieved was revealed postoperatively for each patient via EEG records, and no GTC attacks were recorded. Thus, this limited RFTC procedure provided a relatively safe, precise, and effective treatment for patients with drug-resistant MTLE. Apart from the seizure freedom rate, however, functional outcome is another important issue for patients with a seizure focus originating from the hippocampus, which plays an important role in memory and intelligence functions. Previous reports ${ }^{14,15}$ have shown that neuropsychological measures of memory and IQ significantly improved 2 years following stereotactic radiofrequency ablation, but no improvement was noted after resective operations. The better functional outcome with radiofrequency ablation may be related to preserving some of the mesial temporal structure, such as the parahippocampal gyrus, as well as sparing of the lateral temporal neocortex. ${ }^{9}$ Thus, RFTC for drug-resistant MTLE patients seems to have a similar seizure control outcome but a better functional preservation rate than radiofrequency ablation.

In addition to memory outcome differences, more permanent or transient adverse events or complications following resective operations have been reported, such as stroke, verbal memory decline or loss, wound infections, visual field defects, and depression..$^{8,19,22}$ In our report, no immediate or late adverse events or complications related to the operation were recorded during follow-up. Only three patients had brief periods of minor seizure attacks during the thermocoagulation procedure, which ceased immediately after sedation. Because of the limited wound incision and the relatively limited destruction of brain parenchymal tissue, a lower complication rate is noted in patients undergoing RFTC operations compared to that following resective operations. In reviewing two different studies, we found that RFTC performed at various locations appeared to be safe, showing transient postprocedure side effects at a rate of $2.4 \%$ and permanent neurological deficits in $2.2 \%$ and $1.2 \%$ of the patients, respectively. ${ }^{1,3}$ However, we found no adverse events or complications fol- 


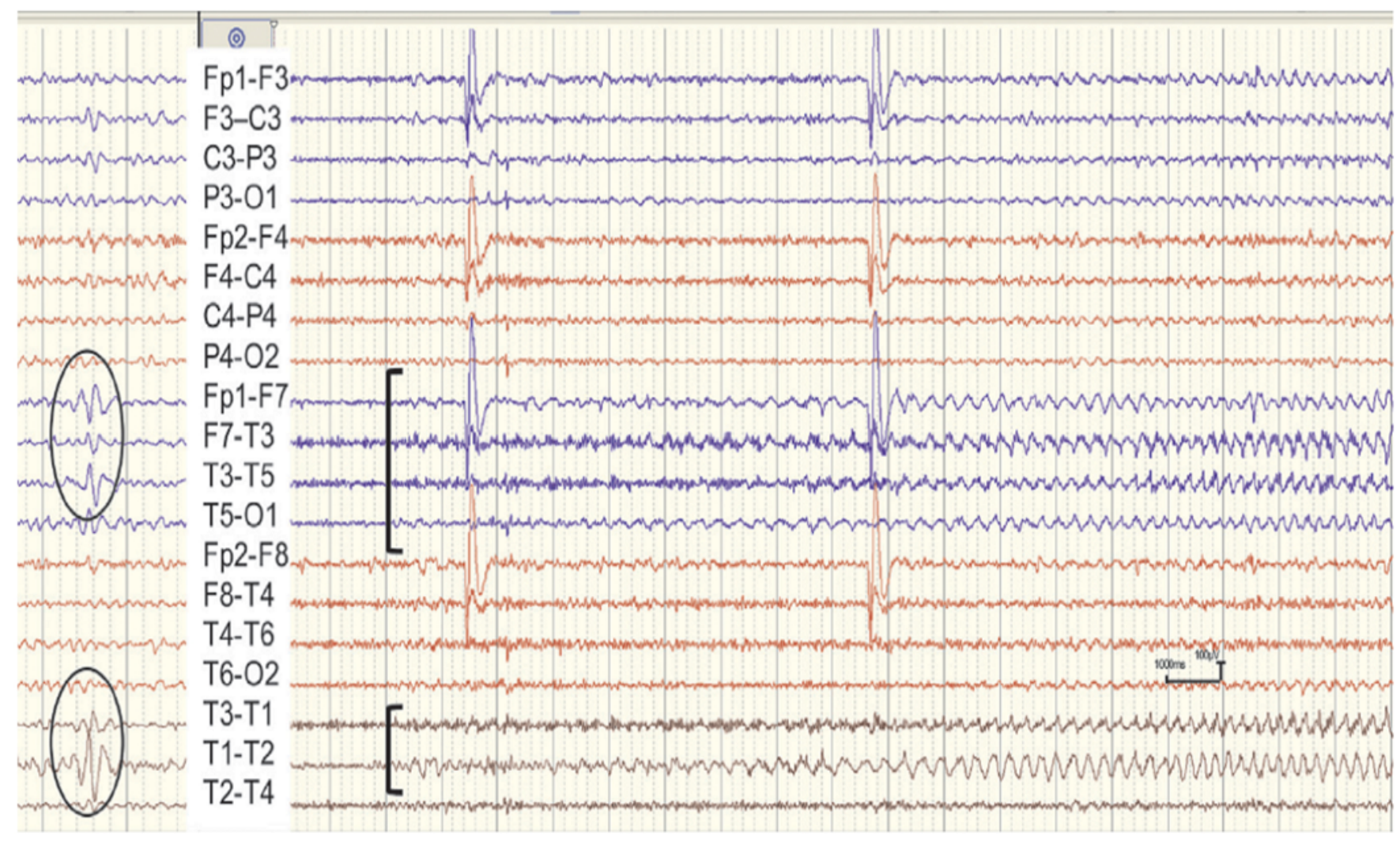

FIG. 3. Case 1. Preoperative EEG shows interictal F7-T1 phase reversal (circles). One episode of CPS with the second GTC seizure is demonstrated, with the corresponding EEG showing initial F7-T1 rhythmic theta activity and propagation to the left frontotemporal region (brackets). Reprinted from World Neurosurgery, 96, Li et al., Seizure freedom after limited hippocampal radiofrequency thermocoagulation, 612.e21-612.e25, Copyright 2016, with permission from Elsevier. Figure is available in color online only.

lowing limited RFTC at the hippocampus. The possible reasons for the lower complication rate and better preservation of memory function in our study may be related to the limited extent of thermocoagulation (lesioned/ nonlesioned volume: $18.7 \%$ on average) and reduction in hippocampal volume loss, including that in the dominant hemisphere, thus leading to the prevention of cognitive impairment and the seizure freedom attained in our patients.

In contrast to previous reports on RFTC at various locations, ${ }^{1-4,10}$ in which perilesional edema was obvious on follow-up MRI even 1-3 months after the procedure, our report showed that the area of perilesional edema was very limited at the immediate postoperative MRI follow-up and that the lesion became smaller on MRI at the 3-month follow-up. The major differences may be explained by several possible factors. First, the previously reported procedures of SEEG-guided RFTC involve first the SEEG electrode implantation and then the RFTC 1-3 weeks later, whereas the recording and RFTC procedures in our study were performed during the same operative period to reduce possible lead displacement due to brain shift or other movement-related displacement. Second, we used a Radionics lead for thermocoagulation, whereas other researchers have used the SEEG leads directly for the RFTC. ${ }^{2-4}$ During our procedure, depth electrodes were applied first for recording the signal of abnormal discharges to identify the location of the possible seizure onset zone and then switched immediately to a Radionics electrode for target depth and trajectory in the RFTC. In our report, we showed two advantages of immediate recording using leads with stylets, followed by RFTC with Radionics electrodes. First, because of the limited time required for the procedure and the obviated need for a permanent or temporary implantation after the procedure, the length of the hospital stay, infection rate, and possible complication rate are minimized, and the related lead displacement or brain shift can also be minimized. To enhance the likelihood of intraoperative recording of epileptic discharges, preoperative withholding of AEDs for 12 hours is essential. If patients continue taking AEDs as usual, the epileptic discharge may not be successfully recorded during surgery. However, withholding AEDs may increase the intraoperative seizure attack but without causing any complications, as observed in three of our patients with minor intraoperative seizures, which were stopped by sedative medications. Second, using Radionics electrodes made of metal materials permits no deformation after heating up to $80^{\circ} \mathrm{C}$. The depth electrodes used in previous series for both SEEG and RFTC have both metal and silicone components, and under a temperature up to $80^{\circ} \mathrm{C}$, the contact part of the two different materials may show deformation, which could then change the curve of the original linear 


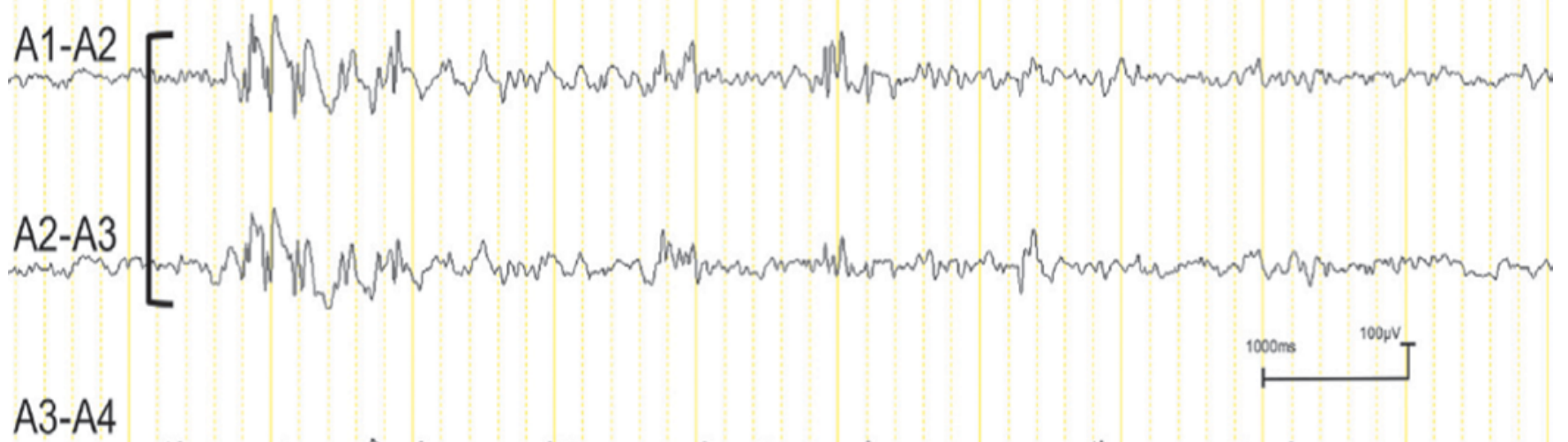

FIG. 4. Case 1. Intraoperative EEG recording with the location of possible seizure foci identified. Intraoperative EEG activity recorded from deep electrodes in the left hippocampus (bracket) demonstrates spike-and-wave complexes over contacts A1-A2 and A2-A3. The patient had the habitual aura sensation during electrical stimulation. Reprinted from World Neurosurgery, 96, Li et al., Seizure freedom after limited hippocampal radiofrequency thermocoagulation, 612.e21-612.e25, Copyright 2016, with permission from Elsevier. Figure is available in color online only.

trajectory. Thus, the larger perilesional edema may have resulted from an altered electrode shape or trajectory due to heat-induced deformation causing parenchymal damage. In comparison to other surgeries, RFTC using depth electrodes to detect the seizure focus and then Radionics electrodes to perform thermocoagulation may show similar outcomes or seizure reduction rates but less edema or parenchymal damage. Moreover, all procedures in our study were performed within 1 hour; therefore, the displacement between depth electrodes and Radionics electrodes was almost impossible to detect with immediate postoperative MRI. Compared with RFTC performed in other series, the sequence of our procedure can produce even less destructive outcomes.

The limitation of this study is its small sample size of only 7 patients. A larger, ongoing series with more patients and a longer follow-up may be more convincing in confirming the efficacy of this procedure.

\section{Conclusions}

Open resection has been a standard surgical treatment for epilepsy for many decades, achieving excellent results in well-selected groups of epilepsy patients, in particular, those with MTLE. However, limited RFTC in a single operation can provide an easier and safer approach in terms of a minimally invasive surgery, resulting in a seizure frequency control rate similar to that observed following open resections. In addition, limited RFTC has unique value given its comparatively low cost and ease of incorporation by different hospitals with various resources. Thus, for patients with drug-resistant unilateral MTLE, RFTC should be considered as one of the treatment choices for its greater safety, lower complication rate, and relatively low healthcare utilization by being more efficient and requiring shorter and less-demanding hospital admissions. Lastly, electrical neuromodulation techniques also hold great promise for minimizing neurocognitive impact while achieving important suppression of epileptic seizures.

\section{Acknowledgments}

We express gratitude to Ms. Mi Mi Chen for her assistance in proofreading the manuscript.

\section{References}

1. Bourdillon $\mathrm{P}$, Isnard J, Catenoix H, Montavont A, Rheims $\mathrm{S}$, Ryvlin P, et al: Stereo electroencephalography-guided radiofrequency thermocoagulation (SEEG-guided RF-TC) in drug-resistant focal epilepsy: results from a 10-year experience. Epilepsia 58:85-93, 2017

2. Cossu M, Cardinale F, Casaceli G, Castana L, Consales A, D'Orio P, et al: Stereo-EEG-guided radiofrequency thermocoagulations. Epilepsia 58 (Suppl 1):66-72, 2017

3. Cossu M, Fuschillo D, Casaceli G, Pelliccia V, Castana L, Mai R, et al: Stereoelectroencephalography-guided radiofrequency thermocoagulation in the epileptogenic zone: a retrospective study on 89 cases. J Neurosurg 123:1358-1367, 2015

4. Dimova P, de Palma L, Job-Chapron AS, Minotti L, Hoffmann D, Kahane P: Radiofrequency thermocoagulation of the seizure-onset zone during stereoelectroencephalography. Epilepsia 58:381-392, 2017

5. Engel J Jr: Mesial temporal lobe epilepsy: what have we learned? Neuroscientist 7:340-352, 2001

6. Engel J Jr: Update on surgical treatment of the epilepsies. Summary of the Second International Palm Desert Conference on the Surgical Treatment of the Epilepsies (1992). Neurology 43:1612-1617, 1993

7. Engel J Jr, McDermott MP, Wiebe S, Langfitt JT, Stern JM, Dewar S, et al: Early surgical therapy for drug-resistant temporal lobe epilepsy: a randomized trial. JAMA 307:922-930, 2012

8. Gleissner U, Helmstaedter C, Schramm J, Elger CE: Memory outcome after selective amygdalohippocampectomy in patients with temporal lobe epilepsy: one-year follow-up. Epilepsia 45:960-962, 2004

9. Gross RE, Mahmoudi B, Riley JP: Less is more: novel lessinvasive surgical techniques for mesial temporal lobe epilepsy that minimize cognitive impairment. Curr Opin Neurol 28:182-191, 2015

10. Guénot M, Isnard J, Ryvlin P, Fischer C, Mauguière F, Sindou M: SEEG-guided RF thermocoagulation of epileptic 
foci: feasibility, safety, and preliminary results. Epilepsia 45:1368-1374, 2004

11. Josephson CB, Dykeman J, Fiest KM, Liu X, Sadler RM, Jette N, et al: Systematic review and meta-analysis of standard vs selective temporal lobe epilepsy surgery. Neurology 80:1669-1676, 2013

12. Kwan P, Brodie MJ: Early identification of refractory epilepsy. N Engl J Med 342:314-319, 2000

13. Li HT, Lee CY, Lim SN, Chang CW, Lee ST, Wu T: Seizure freedom after limited hippocampal radiofrequency thermocoagulation. World Neurosurg 96:612.e21-612.e25, 2016

14. Malikova H, Kramska L, Vojtech Z, Liscak R, Sroubek J, Lukavsky J, et al: Different surgical approaches for mesial temporal epilepsy: resection extent, seizure, and neuropsychological outcomes. Stereotact Funct Neurosurg 92:372-380, 2014

15. Malikova H, Kramska L, Vojtech Z, Lukavsky J, Liscak R: Stereotactic radiofrequency amygdalohippocampectomy: two years of good neuropsychological outcomes. Epilepsy Res 106:423-432, 2013

16. Quigg M, Harden C: Minimally invasive techniques for epilepsy surgery: stereotactic radiosurgery and other technologies. J Neurosurg 121 Suppl:232-240, 2014

17. Sass KJ, Sass A, Westerveld M, Lencz T, Novelly RA, Kim $\mathrm{JH}$, et al: Specificity in the correlation of verbal memory and hippocampal neuron loss: dissociation of memory, language, and verbal intellectual ability. J Clin Exp Neuropsychol 14:662-672, 1992

18. Stroup E, Langfitt J, Berg M, McDermott M, Pilcher W, Como P: Predicting verbal memory decline following anterior temporal lobectomy (ATL). Neurology 60:1266-1273, 2003
19. Thom M, Mathern GW, Cross JH, Bertram EH: Mesial temporal lobe epilepsy: how do we improve surgical outcome? Ann Neurol 68:424-434, 2010

20. Vojtěch Z, Malíková H, Krámská L, Anýž J, Syrůček M, Zámečník J, et al: Long-term seizure outcome after stereotactic amygdalohippocampectomy. Acta Neurochir (Wien) 156:1529-1537, 2014

21. Wechsler D: WAIS-III: Wechsler Adult Intelligence Scale, ed 3. San Antonio: Psychological Corporation, 1997

22. Wiebe S, Blume WT, Girvin JP, Eliasziw M: A randomized, controlled trial of surgery for temporal-lobe epilepsy. $\mathbf{N}$ Engl J Med 345:311-318, 2001

\section{Disclosures}

The authors report no conflict of interest concerning the materials or methods used in this study or the findings specified in this paper.

\section{Author Contributions}

Conception and design: CY Lee. Acquisition of data: all authors. Analysis and interpretation of data: all authors. Drafting the article: CY Lee. Critically revising the article: all authors. Reviewed submitted version of manuscript: all authors. Approved the final version of the manuscript on behalf of all authors: CY Lee. Study supervision: CY Lee.

\section{Correspondence}

Ching-Yi Lee: Chang Gung Memorial Hospital, Taoyuan, Taiwan. 8702021@adm.cgmh.org.tw. 